\title{
Stellar coronagraphy : two mono-axial configurations for the Achromatic Interfero Coronagraph
}

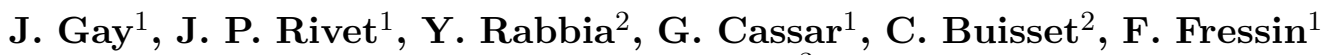 \\ and T. Lepine ${ }^{3}$ \\ ${ }^{1}$ Observatoire de la Côte d'Azur, B.P. 4229, F-06304 Nice Cedex 4, France. \\ email: gay@obs-nice.fr \\ ${ }^{2}$ Observatoire de la Côte d'Azur, Avenue Copernic, F-06130 Grasse, France. \\ ${ }^{3}$ Ecole Supérieure d'Optique, LTSI, 10 rue Barrouin, F-42000 Saint-Etienne, France
}

\begin{abstract}
The standard AIC (Achromatic Interfero Coronagraph) has a "coudé" geometry (the output beam leaves at right angle from the input beam). Thus, some extra optical parts are required to fit such a device within the optical train between a telescope and its IR camera. To avoid this drawback, we present two mono-axial variants of the AIC.
\end{abstract}

Keywords. instrumentation: miscellaneous.

\section{Basics on the standard AIC}

The Achromatic Interfero-Coronagraph (AIC) has been introduced by Gay \& Rabbia (1996) (see also Baudoz et al., 2000) as a new concept of stellar coronagraphs. Instead of rejecting the light of a central star by a more or less smoothly varying complex transmission in the focal plane of a telescope, the AIC rejects the light by destructive interferences. The device works like a modified Michelson's interferometer (see Figure 1): the input light is divided by a beam splitter cube into two balanced beams leaving at right angle. The first one passes through the "passive" arm (thick solid lines on Figure 1), which involves only flat mirrors. The second one enters the "phase shifting" arm (thick dashed lines on Figure 1). The latter arm involves a cat's eye structure with a primary parabolic mirror and a small secondary spherical mirror, so that the light beam passes through a focus. Crossing a focal point is known to lead to an achromatic $\pi$ phase shift for the amplitude (see Gouy, 1890 and Born \& Wolf, 1999), and a central symmetry of the image. So, when both beams recombine, the light of a star lying on the axis (thus invariant by central symmetry) cancels because of the $\pi$ phase shift, provided both arms of the interferometer have equal optical length. Cancellation does not occur for off-axis sources.

Being a Michelson's interferometer, the AIC has a "coudé" structure: the output and input beams are orthogonal. Thus, some light-, time- and space-consuming optical components need to be added in order to insert the AIC between an adaptative optics device and the camera of an existing telescope (see Figure 2 for a view of those extra components, in the CFHT implementation).

To avoid these bulky extra components, we propose two new optical combinations: the "CIAXE" and the "open-air CIAXE" or "CIAXA". Both are based upon the same principle of interferometric nulling as the standard AIC, but with a fully mono-axial and very compact design, so as to make them more "plug-and-play" (or "insert-and-see"). 


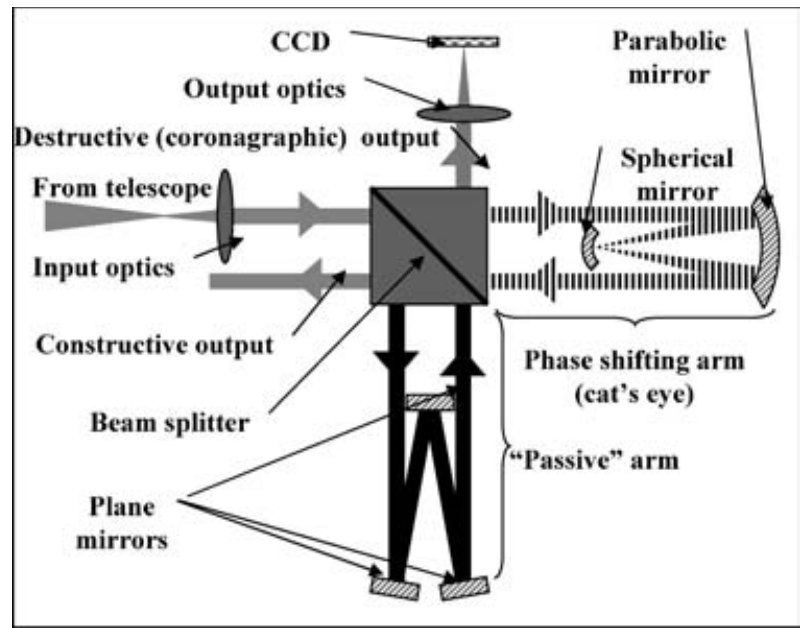

Figure 1. The configuration of the standard AIC.

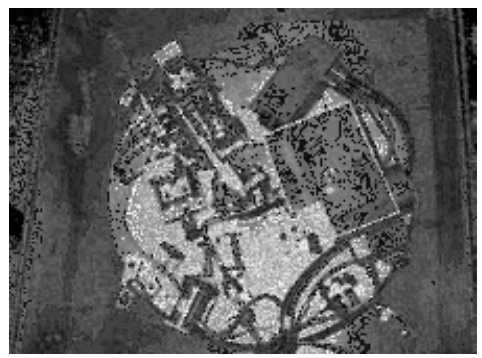

Figure 2. The standard AIC with extra optical components designed to fit it within the $3.6 \mathrm{~m}$ telescope of CFH (Hawaii), between the adaptative optics PUEO and the IR camera KIR. This is what CIAXE avoids!

\section{The CIAXE: a mono-axial version of the AIC}

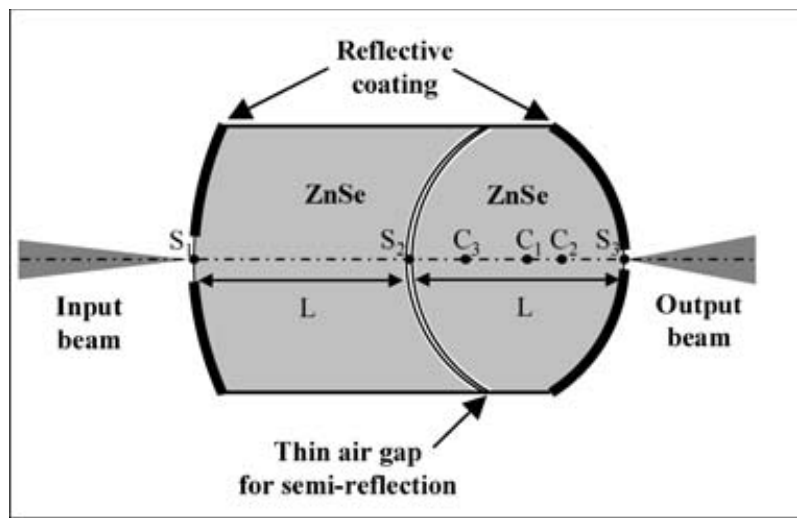

Figure 3. The configuration of the CIAXE.

The first and simplest of these two mono-axial alternatives to the AIC is sketched in Figure 3. It is based upon two equally thick lenses (made out of $Z n S e$ for example), which are assembled together with a calibrated spacer ring, so as to leave between them 
a thin air gap. This air gap is chosen and possibly coated so as to act as a balanced beam splitter over the desired bandwidth. For optimal rejection, the thicknesses of both lenses have to be equal to the best possible precision (a difference of $20 \mathrm{~nm}$ limits the rejection to 1000 in $K$ band). The external surfaces have to be fully reflecting, except for two small circular zones surrounding the axis, so as to let the input and output beams enter and leave the CIAXE.

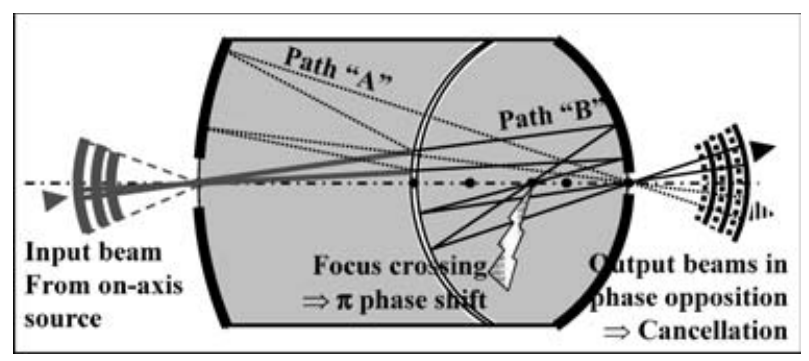

Figure 4. Ray tracing through the CIAXE for an on-axis source.

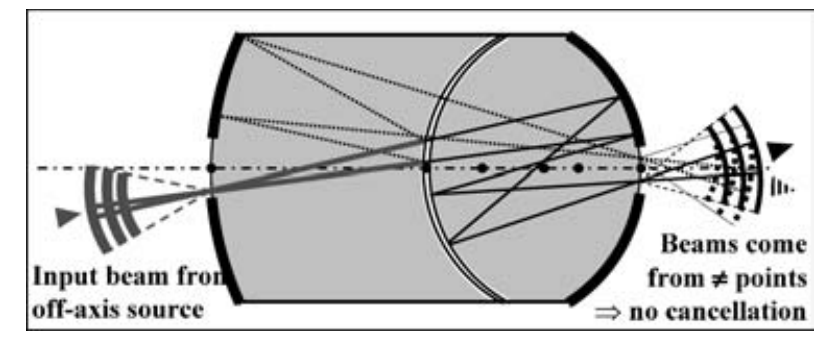

Figure 5. Ray tracing through the CIAXE for an off-axis source.

Figures 4 and 5 show the typical optical paths within the CIAXE, for a light ray coming from an on-axis source, or from an off-axis source. The constraints to be fulfilled by the three radii of curvature are (Gay et al., 2005):

- The image of $S_{1}$ must be $S_{3}$, through Paths $A$ and $B$.

- The magnifications along Paths $A$ and $B$ must be opposite.

- The optical paths $A$ and $B$ must have equal length.

- Path $B$ must cross a focus, and Path $A$ must not.

Given the geometric thickness $L$, the solution is unique:

$$
\left\{\begin{array}{l}
R_{1}=L \frac{4}{4-\sqrt{2}} \\
R_{2}=L \frac{1}{\sqrt{2}}, \\
R_{3}=L \frac{-4}{4+\sqrt{2}}
\end{array}\right.
$$

The light along Path $A$ reaches the exit aperture around $S_{3}$ without crossing a focus. On the contrary, the light along Path $B$ does cross a focus : it is achromatically phaseshifted of $\pi$. Thus, when recombining, the light from an on-axis source cancels (if the telescope pupil is axis-symmetric, and provided the optical paths be equal).

For an off-axis source, the lights along Paths $A$ and $B$ converge onto two different (symmetric) points of the exist aperture. Thus no cancellation occurs. 
It can be shown (Gay et al., 2005) that for an ideally working device, all the unwanted light of the central source is sent back to the entrance by successive reflections and transmissions on the beam-splitter gap.

\section{The CIAXA, an "open-air" version of the CIAXE}

As described above, the CIAXE has a drawback: the light travels in Zinc Selenide, which is a high refraction index medium (index above 2.4 in $K$ band). Thus the wavelength is noticeably shorter than in the air. This leads to constraints on the optical surfaces accuracy which happen to be slightly above the capabilities of diamond turning techniques. Thus, an "open-air" version of the CIAXE, the "CIAXA" (for CIAXE + Air) has been designed, where the light essentially travels in air (see Figure 6).

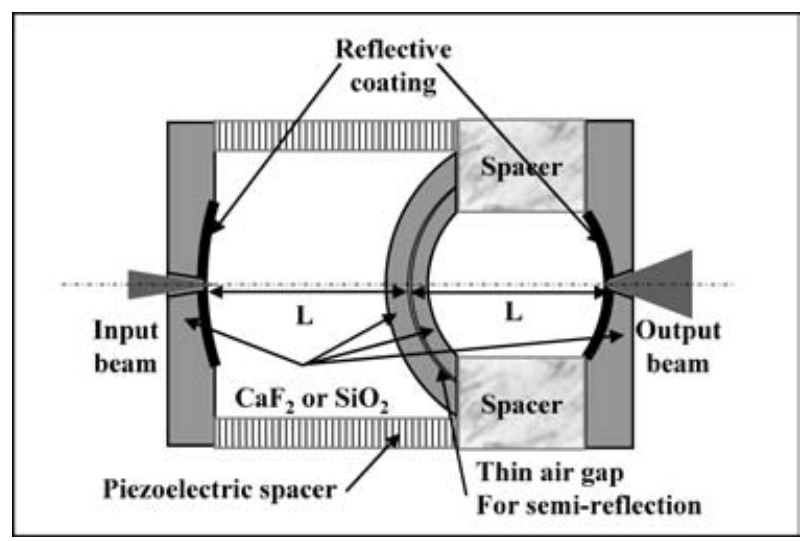

Figure 6. The "CIAXA", an open-air version of the CIAXE.

The piezoelectric spacers are needed to provide for an accurate fine tuning of the optical path difference. This is both an advantage (the accuracy less depends on machining efficiency), and a drawback (the device is more complex, and thus less autonomous).

\section{Conclusion}

The CIAXE and CIAXA work the same way as the standard AIC: they do stellar coronagraphy by destructive interferences between two balanced fractions of the input beam, one of which being achromatically $\pi$ phase-shifted by focus crossing.

The specificity of the two variants under consideration is their easy integration in existing telescopes, due to their mono-axial structure. Some R\&D effort is in progress to find machining procedures that meet the rather severe accuracy requirements of these components.

\section{References}

Gay, J. \& Rabbia, Y. 1996, C.R. Acad. Sci. Paris II 322, 265

Baudoz, P., Rabbia, Y., \& Gay, J. 2000, Astr. Astroph. Suppl. Series 141, 319

Gouy, L.G. 1890, C.R. Acad. Sci. Paris 110, 1251

Born, M. \& Wolf, E. 1999, (Cambridge University Press), p. 499

Gay, J., Fressin, F., Rivet, J.P., Rabbia, Y., \& Buisset, C. 2005, C.R. Acad. Sci Paris II, submitted. 